\title{
A Participatory Return-to-Work Intervention for Temporary Agency Workers and Unemployed Workers Sick-Listed Due to Musculoskeletal Disorders: Results of a Randomized Controlled Trial
}

\author{
Sylvia J. Vermeulen $\cdot$ Johannes R. Anema $\cdot$ \\ Antonius J. M. Schellart • Dirk L. Knol • \\ Willem van Mechelen • Allard J. van der Beek \\ Published online: 19 February 2011 \\ (c) The Author(s) 2011. This article is published with open access at Springerlink.com
}

\begin{abstract}
Introduction Within the labour force workers without an employment contract represent a vulnerable group. In most cases, when sick-listed, these workers have no workplace/employer to return to. Therefore, the aim of this study was to evaluate the effectiveness on returnto-work of a participatory return-to-work program compared to usual care for unemployed workers and temporary agency workers, sick-listed due to musculoskeletal disorders. Methods The workers, sick-listed for 2-8 weeks due to musculoskeletal disorders, were randomly allocated to the participatory return-to-work program $(n=79)$ or to
\end{abstract}

Trial registration: The Netherlands Trial Register (NTR); NTR1047.

S. J. Vermeulen · J. R. Anema (凹) - A. J. M. Schellart .

W. van Mechelen · A. J. van der Beek

Department of Public and Occupational Health, EMGO Institute for Health and Care Research, VU University Medical Centre, P.O. Box 7057, 1007 MB Amsterdam, The Netherlands

e-mail: h.anema@vumc.nl

\section{S. J. Vermeulen}

e-mail: s.vermeulen@vumc.nl

A. J. M. Schellart

e-mail: ton.schellart@vumc.nl

W. van Mechelen

e-mail: w.vanmechelen@vumc.nl

A. J. van der Beek

e-mail: a.vanderbeek@vumc.nl

S. J. Vermeulen · J. R. Anema · A. J. M. Schellart .

W. van Mechelen · A. J. van der Beek

Research Centre for Insurance Medicine AMC-UMCG-UWV-

VUmc, Amsterdam, The Netherlands

D. L. Knol

Department of Epidemiology and Biostatistics, VU University

Medical Centre, Amsterdam, The Netherlands

e-mail:d.knol@vumc.nl usual care $(n=84)$. The new program is a stepwise procedure aimed at making a consensus-based return-to-work plan, with the possibility of a temporary (therapeutic) workplace. Outcomes were measured at baseline, 3, 6, 9 and 12 months. The primary outcome measure was time to sustainable first return-to-work. Secondary outcome measures were duration of sickness benefit, functional status, pain intensity, and perceived health. Results The median duration until sustainable first return-to-work was 161 days in the intervention group, compared to 299 days in the usual care group. The new return-to-work program resulted in a non-significant delay in RTW during the first 90 days, followed by a significant advantage in RTW rate after 90 days (hazard ratio of 2.24 [95\% confidence interval 1.28-3.94] $P=0.005$ ). No significant differences were found for the measured secondary outcomes. Conclusions The newly developed participatory return-to-work program seems to be a promising intervention to facilitate work resumption and reduce work disability among temporary agency workers and unemployed workers, sick-listed due to musculoskeletal disorders.

Keywords Work disability - Return-to-work interventions · Musculoskeletal disorders - Vulnerable worker populations - Worker without employment contract

\section{Introduction}

Sickness absence and work disability are a common and substantial public health problem with major economical consequences worldwide [1, 2]. Given the fact that longterm sickness absence contributes largely to the total amount of annual work disability costs in Western countries [1], development of effective return-to-work (RTW) 
interventions are considered important public health (research) challenges [3].

To date, most RTW intervention research is aimed at sicklisted (established regular) employees, i.e. workers with relatively permanent employment relationships. In contrast, development of effective RTW interventions for sick-listed workers without an employment contract is lagging [4, 5]. However, in view of the growing international trend towards labour market flexibility [6], development of RTW interventions specifically aimed at sick-listed workers without an employment contract and sick-listed workers with a flexible labour arrangement, e.g. temporary agency workers, is of crucial importance. These workers represent a vulnerable group within the working population. Various studies show a poorer health status and an increased risk for (long-term) work disability among these workers, compared to regular employees [7-12]. In addition, they are burdened with a greater distance to the labour market $[11,13,14]$. When sicklisted, these workers have in most cases no workplace/ employer to return to $[15,16]$. Hence, tailor-made RTW interventions with the presence of a workplace for (therapeutic) RTW could be an important factor in the recovery and (vocational) rehabilitation process [15]. Therefore, a participatory RTW program was developed based on a successful RTW intervention for regular employees, sick-listed due to low back pain $[17,18]$. This newly developed RTW program comprises of a stepwise communication process to identify and solve obstacles for RTW, resulting in a consensus-based plan to facilitate (therapeutic) RTW. The three main stakeholders in this intervention are: the sick-listed worker, the labour expert representing the Social Security Agency (SSA) who guides the worker with regard to vocational rehabilitation, and an independent RTW coordinator. The role of the RTW coordinator is to stimulate a high degree of involvement of both the sick-listed worker and the labour expert, and to reach consensus about the RTW plan. To offer a workplace for (therapeutic) RTW, a vocational rehabilitation agency was contracted to find a suitable (therapeutic) workplace matching with the formulated RTW plan.

The aim of this study was to assess the effectiveness of the new participatory RTW program compared to usual care for unemployed workers and temporary agency workers, sicklisted due to musculoskeletal disorders (MSD). The primary outcome measure was time to sustainable first RTW. Duration of sickness benefit was secondary outcome measure.

\section{Methods}

\section{Study Design and Setting}

The study is a randomized controlled trial carried out in collaboration with five front offices of the Dutch National
Social Security Agency (SSA) and four large Dutch commercially operating vocational rehabilitation agencies (Olympia, Adeux, Capability, and Randstad Rentrée) in the eastern part of the Netherlands. The Medical Ethics Committee of the VU University Medical Centre (Amsterdam, the Netherlands) approved the study design, the protocols and procedures, and informed consent. The design of the study has been described in detail elsewhere [19].

\section{Study Population and Recruitment}

Between March 2007 and September 2008 all temporary agency workers and unemployed workers who were sicklisted between one and 2 weeks due to MSD and lived in the eastern part of the Netherlands received a letter with a screening questionnaire from the insurance physician of the SSA, on behalf of the researchers. The workers who returned the screening questionnaire indicating that they were still sick-listed and interested in participation, were contacted by the researchers by telephone to give additional information about the content of the study and to check eligibility. Temporary agency workers and unemployed workers sick-listed between 2 and 8 weeks with MSD as main health complaint for their sickness benefit claim were included. The main exclusion criteria were: (1) being sick-listed for more than 8 weeks; (2) not being able to complete questionnaires written in the Dutch language; (3) having a conflict with the Social Security Agency regarding a sickness benefit claim or a long-term disability claim; (4) having a legal conflict, e.g. an ongoing injury compensation claim; and (5) having had an episode of sickness absence due to MSD within 1 month before the current sickness benefit claim.

The insurance physician of the SSA was responsible for the identification of severe co-morbidity among the included workers; i.e. having a terminal disease, having a serious psychiatric disorder, or having a serious cardiovascular disease. These participants remained in the intervention group, but were excluded from the participatory RTW program.

\section{Randomization and Blinding}

Before randomization, to prevent unequal distribution of relevant prognostic baseline characteristics, the sick-listed workers were pre-stratified based on two important prognostic factors, namely type of worker [20-22], i.e. temporary agency worker or unemployed worker, and degree of mental or physical work demands (light or heavy) in last job held before the current sickness benefit claim [23, 24]. Next, block randomization (using blocks of four allocations) was applied to ensure equal group sizes within each stratum. A separate block randomization table was generated for each 
of the five participating SSA front offices. Allocation to the intervention group or the usual care group was performed after informed consent and completion of the baseline questionnaire.

The participants and occupational health care professionals were not blinded to the allocation result. Data regarding work resumption and sickness benefit claim duration were collected from the SSA database. Data entry of the self-reported data was performed by a research assistant using a unique research code for each participant, to ensure that analyses of the data by the researcher was blinded.

\section{Interventions}

\section{Usual Care}

In the Netherlands, workers who are sick-listed and who have no (longer) an employment contract, i.e. no employer/ workplace to return to, are entitled to supportive income and occupational health care by the SSA during his/her sickness benefit period. Vocational rehabilitation is carried out by a team of occupational health care professionals from the SSA, consisting of an insurance physician, a labour expert, and a case-manager. The insurance physician of the SSA guides the worker according to the guidelines for occupational health care of the Netherlands Society of Occupational Medicine. He/she advises about recovery, e.g. health promotion and RTW options, and, if necessary, he/she can advise and refer to work disability oriented treatment/guidance, such as graded physical therapy or work-related psychological help. The labour expert is responsible for vocational rehabilitation support. Based on a personal examination of the work abilities of the worker (including the problem analysis performed by the insurance physician) and expert knowledge of the (regional) labour market, the labour expert advises the worker with respect to return-to-work options. When the chance of work resumption in regular work without additional vocational rehabilitation support is viewed as slim, interventions such as referral to a vocational rehabilitation agency, personal coaching or short-term education/training are offered to the worker. The case manager of the SSA monitors the vocational rehabilitation process and regularly keeps in contact with the worker to evaluate the progress. In case of an impeded (vocational) recovery/rehabilitation process the case manager consults with, and if necessary refers to, the insurance physician or the labour expert to identify and tackle the cause of this stagnation. This can lead to alterations in the vocational rehabilitation guidance, for instance offering more intensive personal guidance or referral to a graded activity program. The occupational health care by the SSA ends when the sickness benefit ends, i.e. when full recovery of health is present and/or when full recovery of work ability is established by the insurance physician. Both can occur without actual RTW of the worker.

\section{Participatory RTW Program}

The intervention group received usual care. This did not differ from the vocational rehabilitation guidance offered to the workers in the usual care group, i.e. the earlier described roles of the OHC professionals. However, in addition, these sick-listed workers were referred by their insurance physician to a RTW coordinator for the new participatory RTW program. The aim of this new program was to make a consensus-based RTW plan. In this study the RTW coordinator was an employee of the SSA, in most cases with a labour expert background, with experience in process guidance, with sufficient knowledge and experience regarding (vocational) rehabilitation, and no involvement in the usual care guidance of the sick-listed worker to guarantee independency. All RTW coordinators received training prior to the start of the study.

The newly developed RTW program consisted of consecutive steps starting with a combined consult with the insurance physician and the labour expert of the SSA. Next, two structured meetings took place between the sicklisted worker and the RTW coordinator, and between the labour expert of the SSA and the RTW coordinator, respectively. In the meeting with the sick-listed worker the RTW coordinator used a structured interview to identify and prioritise obstacles for RTW. The ranking of identified obstacles for RTW was performed based on frequency (how often do they occur?) and severity (how large is the perceived impact on functioning in daily life and/or work?). The meeting between the RTW coordinator and the labour expert was carried out in a comparable manner and resulted in a selection of prioritised obstacles for RTW from the perspective of the labour expert. Next, the RTW coordinator, the sick-listed worker, and the labour expert brainstormed about solutions to address the prioritised obstacles. The proposed solutions were judged on the basis of availability, feasibility and ability to solve the barrier. The final step resulted in the making of a consensus-based RTW plan describing the prioritised obstacles for RTW, the consensus-based solutions, the person(s) responsible for implementation of each selected solution, and a time-path when it should be carried out. Furthermore, to create a possibility for therapeutic work resumption, a commercially operating vocational rehabilitation agency could be contracted to find a temporary (therapeutic) workplace matching with the formulated RTW plan and taking into account the worker's (functional) limitations. Six weeks after the brainstorm session the RTW coordinator 
contacted the sick-listed worker and the labour expert by telephone to evaluate actual implementation of the solutions, including the progress regarding placement in temporary (therapeutic) work. A more detailed content of the structured meetings with the RTW coordinator is presented in Table 1. The content of the entire new participatory RTW program has been described in detail elsewhere [15].

Table 1 Content of the structured meeting with the RTW coordinator

Content of the structured meeting with the RTW coordinator

Introduction

Check if the worker, the insurance physician and the labour expert agree with following the participatory program.

Explain the independent role of the RTW coordinator.

Explain that the main goal is to make a consensus based RTW plan. Inventory of obstacles for RTW

Meeting with the worker

Starting point is the inventory of obstacles for RTW given by the insurance physician as home assignment to the worker after the first consult.

Identify (perceived) work- and non-work related obstacles for RTW from the perspective of the worker. Use the following categories as a framework: personal factors, social factors, physical environment demands (e.g. ergonomic obstacles at the workplace), dynamic action demands (e.g. repetitive work), static posture demands, work experience, commuting, remaining factors (e.g. financial problems).

Rank the identified obstacles based on frequency and perceived severity.

Meeting with the labour expert

Identify (perceived) work- and non-work related obstacles for RTW from the perspective of the labour expert.

Rank the identified obstacles based on frequency and perceived severity.

Brainstorm session with the worker and the labour expert

The 3 top ranked obstacles for RTW from both the worker and the labour expert are the starting point.

Think of solutions for all 6 prioritised obstacles, e.g. reduction of physical workload, graded return-to-work, improving the commuting distance, short-term education, help with dept repayment.

Stimulate active involvement from the worker and the labour expert.

Choose solutions based on availability, feasibility and ability to solve the obstacle.

Making of the consensus-based RTW plan

Give a summary of the prioritised obstacles for RTW, the chosen (consensus based) solutions, if possible a concrete work(place) profile, the person(s) responsible for implementation of the solution(s), and a time-path.

Underline the importance of own initiative of the worker to achieve RTW.

Sent the report to the worker, the labour expert, and the insurance physician.

If chosen for finding a suitable temporary (therapeutic) workplace, contact the case manager of the contracted vocational rehabilitation agency.
Outcome Measures

\section{Data Collection}

Prior to randomization the baseline measurement was performed. Follow-up measurements took place at 3, 6, 9 and 12 months after baseline. Data regarding RTW were obtained from both the SSA database, including the workers' file, and the self-report questionnaires at 12-months follow-up. Data on sickness benefit were collected from the SSA database. Data regarding applied occupational health care interventions were obtained from the SSA database and the medical file of the worker at the SSA.

\section{Primary Outcome Measure}

The primary outcome measure in this study was sustainable first RTW, which was defined as the duration in calendar days from the day of randomization until first sustainable return-to-work, i.e. return-to-work in any type of paid work or work resumption with ongoing benefits for at least 28 consecutive (calendar) days.

\section{Secondary Outcome Measures}

Secondary outcome measures in the study were duration of sickness benefit, pain intensity, and functional status. Duration of sickness benefit was measured as a separate outcome measure because, contrary to regular employees, for sick-listed temporary agency workers and sick-listed unemployed workers recovery of health and/or functional limitations with ending of the sickness benefit does not necessarily coincide with actual RTW. First sustainable ending of sickness benefit was defined as the duration in calendar days from the day of randomization until ending of sickness benefit for at least 28 days. Recurrence of sickness absence with an accepted sickness benefit claim within 28 days after ending of the previous sickness benefit was considered as belonging to the preceding sickness benefit period, on condition that it was due to the same (or related) MSD. The total number of days of sickness benefit during the entire 12-months follow-up period was also calculated. Musculoskeletal pain intensity was measured using the Von Korff questionnaire [25]. Functional status, i.e. perceived functional impairments in daily life, and general health were assessed with the Dutch translation of the SF-36 [26, 27].

\section{Prognostic Measures}

All covariates were measured at baseline. Type of previous work (light or heavy demanding) and work status (working or not working) directly prior to reporting sick, i.e. before 
the onset of work disability, were collected, since findings in the international literature indicate that both items might be prognostic factors for the duration of sickness absence and work disability [20-22, 24]. Furthermore, behavioural determinants were included in the baseline measurement. Pain coping was assessed with the Pain Coping Inventory Scale (PCI) [28]. Behavioural determinants for RTW consisted of the workers' attitude, social influence, and self-efficacy with regard to RTW, and the workers' intention to RTW despite symptoms due to MSD. The Attitude, Social Influence and self-Efficacy (ASE) determinants were assessed using a questionnaire developed earlier by Van Oostrom and colleagues [29].

\section{Statistical Analyses}

All statistical analyses were carried out at workers' level and according to the intention-to-treat principle. To determine whether randomisation was performed successfully descriptive statistics were used to compare the baseline measurements of both groups. The results of the intentionto-treat analyses were compared to per-protocol analyses to assess the presence of bias due to protocol deviations.

The Kaplan-Meier method was used to describe the duration until sustainable RTW in both groups. The Cox proportional hazard model was used to estimate hazard ratios (HR) for sustainable RTW and the corresponding 95\% confidence intervals. First, unadjusted Cox regression analysis was carried out and, if necessary, adjusted Cox regression analysis was performed to adjust for prognostic dissimilarities at baseline, i.e. a confounder was added to the model when the regression coefficient changed by $10 \%$ or more. To account for clustering of participants within insurance physicians and within the couples of labour experts and RTW coordinators the shared-frailty procedure was used [30]. Linear mixed models were used to assess differences in pain intensity, functional status and perceived health, i.e. the interaction between treatment group and measurement time (baseline, 3, 6 and 12 months), adjusted for baseline differences, and taking into account clustering on the level of the insurance physician. Stata version 11.0 was used to test for clustering in the Cox regression analysis. All other analysis were performed with SPSS version 15.0. For all analyses a $P$ value of 0.05 (twotailed) was considered statistically significant.

\section{Results}

\section{Recruitment of Participants}

Recruitment of participants took place between March 2007 and September 2008. The returned screening questionnaires resulted in 784 potentially eligible workers who were interested in participation. After telephone contact 191 workers refused participation and 327 workers did not meet the inclusion criteria, resulting in 266 workers for whom intake meetings were planned. During the intake meeting 103 workers were not included due to several reasons (see Fig. 1). Finally, 163 workers who met all inclusion criteria were enrolled in the study and randomised to the participatory RTW program $(n=79)$ or usual care $(n=84)$. An overview of the recruitment flow is presented in Fig. 1.

Loss to Follow-Up

Data about RTW and sickness benefit were available for all workers for the whole 12-months follow-up period. The RTW data were collected from the SSA database, including the workers' file, and the self-report questionnaires. Data about sickness benefit were collected from the SSA database. For the self-reported secondary outcomes complete followup data were available for 116 participants $(=71.2 \%)$.

\section{Baseline Characteristics}

Table 2 presents a summary of the measured baseline characteristics of the participants in the participatory RTW program group and the usual care group. For most of the baseline characteristics (i.e. worker-related, pain-related, health-related, work-related, and behavioural determinants) there were no or only minor (non-significant) differences between the two groups. All participants were fully work disabled at the time of enrolment. Approximately half of the workers in both groups (usual care group 52.4\% and intervention group $54.4 \%$, respectively) worked prior to reporting sick, i.e. the onset of work disability. For the participants who did not work before reporting sick the median duration between end of last job and first day of reporting sick was 13.0 months (interquartile range (IQR) 6.3-45.3 months) in the usual care group and 13.5 months (IQR 6.0-43.5 months) in the participatory RTW program group. However, despite randomisation, prognostic dissimilarities were present at baseline with worse physical role functioning $(P=0.052)$; more regular work schedule in last work $(P=0.031)$; and less intention to RTW despite symptoms $(P=0.024)$ in controls. If necessary, for these dissimilarities was adjusted in analyses.

\section{Compliance}

In the usual care group 7 workers did not receive usual care as they reported full recovery of health complaints with subsequent ending of sickness benefit shortly after randomisation. Also 7 workers in the participatory RTW 


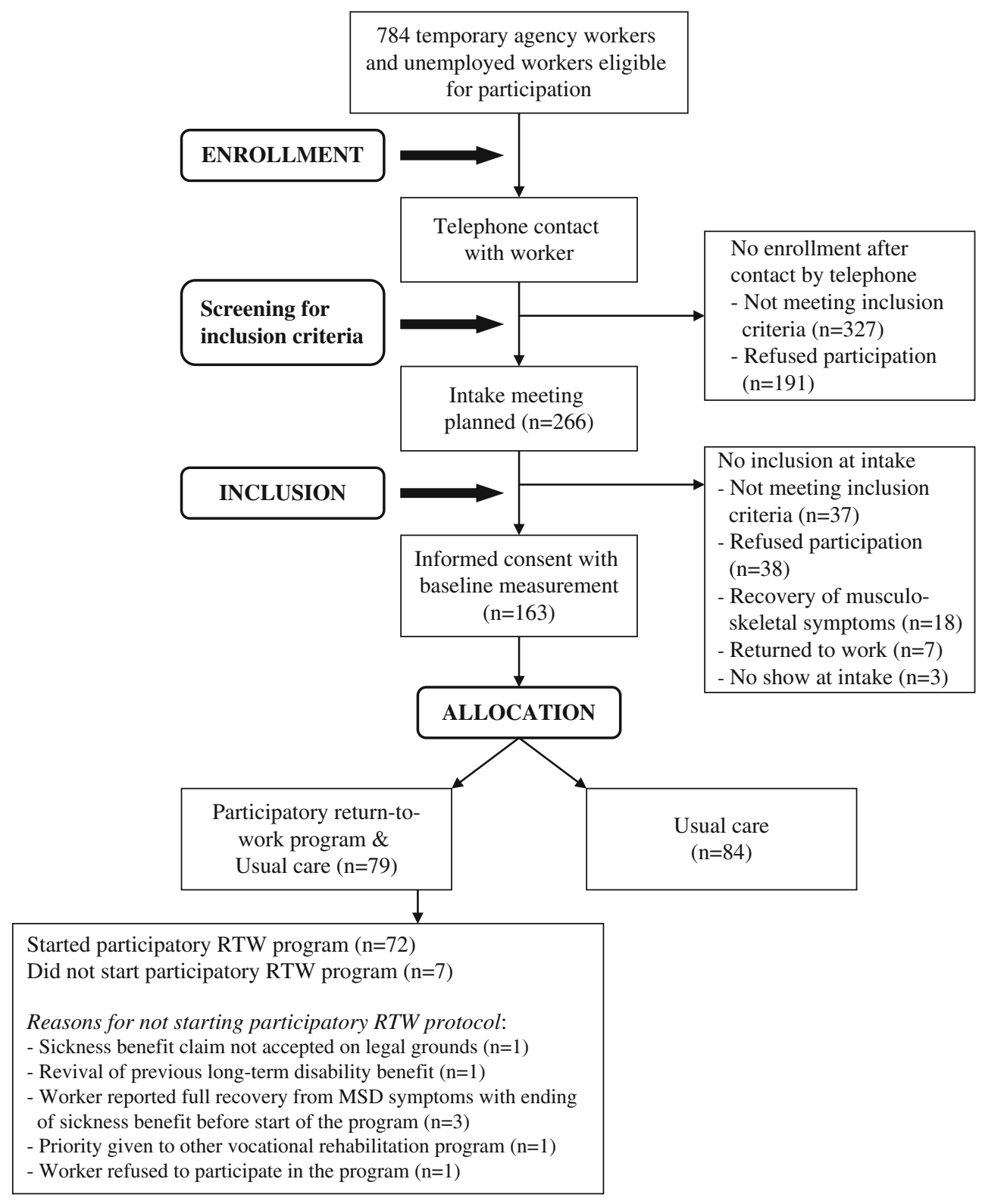

Fig. 1 Flow of the workers in the study

program group did not receive the allocated intervention, i.e. the participatory RTW program was not followed, due to several reasons (see Fig. 1). The remaining 72 workers in the intervention group all had the first consult with the insurance physician. One worker reported full recovery of health with ending of sickness benefit before the meeting with the RTW coordinator. For 23 workers the insurance physician established full work ability with ending of sickness benefit, i.e. claim closure, during the first consult. In case of claim closure without actual RTW, these workers were, in accordance with the usual care policy of the SSA, not referred to the RTW coordinator for making a RTW action plan. In addition, following the protocol, 10 workers were not referred to the RTW coordinator as the insurance physician established absence of work ability on medical grounds for at least 3 months during the first consult. The remaining 38 workers in the intervention group had the meetings with the labour expert and the RTW coordinator with the making of a consensus based RTW plan. Referral to a vocational rehabilitation agency for finding a suitable temporary workplace took place for 30 workers. Placement in a temporary (therapeutic) workplace was successfully achieved for 22 workers. In addition, four workers found a suitable workplace on own initiative. The median duration of working in a temporary (therapeutic) workplace was 90 days (IQR 41-147 days). 
Table 2 Baseline characteristics of the workers without employment contract, sick-listed due to musculoskeletal disorders $(N=163)$

\begin{tabular}{lll}
\hline & $\begin{array}{l}\text { Intervention group } \\
(N=79)\end{array}$ & $\begin{array}{l}\text { Control group } \\
(N=84)\end{array}$ \\
\hline Age (mean \pm SD) & $44.0 \pm 10.7$ & $45.6 \pm 9.0$ \\
Gender (\% male) & 57.0 & 63.1 \\
Level of education (\% low) & 57.0 & 60.7 \\
Pain intensity (1-10 score) (mean \pm SD) & & \\
Back pain & $7.1 \pm 2.0$ & $6.8 \pm 1.9$ \\
Neck pain & $7.1 \pm 1.7$ & $6.7 \pm 2.0$ \\
Other pain & $6.5 \pm 1.8$ & $6.3 \pm 1.9$ \\
Functional status (0-100 score) (mean \pm SD) & & \\
Physical functioning & $46.0 \pm 22.1$ & $51.4 \pm 21.3$ \\
Social functioning & $49.4 \pm 25.4$ & $51.2 \pm 27.5$ \\
Perceived health $(0-100$ score) (mean \pm SD) & $56.3 \pm 21.8$ & $60.0 \pm 20.3$ \\
Type of worker (\%) & & \\
Temporary agency worker & 51.9 & 52.4 \\
Unemployed worker & 48.1 & 47.6 \\
Type of last work (\% physically and/or mentally demanding) & 74.7 & 75.0 \\
Work schedule (\% day work) & 58.2 & 78.3 \\
Worker's expectation regarding RTW at baseline (mean \pm SD) & $2.22 \pm 1.15$ & $2.14 \pm 1.12$ \\
Intention to RTW despite symptoms (1-5) (mean \pm SD) & $3.46 \pm 1.10$ & $3.05 \pm 1.19$ \\
\hline
\end{tabular}

During the 12-months follow-up 12 of the 22 workers with therapeutic work resumption were offered an employment contract.

\section{Usual Care}

\section{Consults with the Occupational Health Care Professionals}

In the participatory RTW program group 21 workers (total of 23 consults) had a consult with the case-manager of the SSA, compared to 41 workers (total of 49 consults) in the usual care group. However, the workers in the participatory RTW program group had more consults with the insurance physician ( $n=70 ; 157$ consults) and the labour expert ( $n=36 ; 55$ consults) of the SSA, compared to the usual care group, where 60 workers (total of 107 consults) reported a consult with the insurance physician and 19 workers (total of 26 consults) reported a consult with the labour expert.

\section{Received Occupational Health Care Interventions}

In the participatory RTW program group 25 workers received a usual care intervention (total of 28 interventions) during follow-up with a median duration of 6.4 months (IQR 3.0-12.4 months), compared to 30 workers in the usual care group (total of 32 interventions) with a median duration of 7.4 months (IQR 2.9-11.2 months). Three workers in the participatory RTW program group and two workers in the usual care group received two occupational health care interventions. The received usual care interventions consisted of: (1) offering (short-term) education/ training (participatory RTW program group (PWP) $n=11$, usual care group (UC) $n=5$ ); (2) referral to a vocational rehabilitation agency (PWP $n=4$, UC $n=9$ ); (3) referral to an employment agency for employment-finding (PWP $n=5$, UC $n=4$ ); (4) personal coaching (PWP $n=3$, UC $n=3$ ); (5) interview training (including writing a job application letter) (PWP $n=2$, UC $n=4)$; (6) placement in a temporary workplace (on trial) (PWP $n=1$, UC $n=$ $0)$; (7) searching for a sheltered workplace (PWP $n=1$, UC $n=3)$, (8) on-the-job training (PWP $n=1$, UC $n=1)$; (9) referral to a graded activity program (PWP $n=0$, UC $n=2)$; and (10) type of intervention unknown ( $\operatorname{PWP} n=0$, $\mathrm{UC} n=1)$.

\section{Return-to-Work}

The median time until sustainable first RTW was 161 days (IQR 88-365 days) in the participatory RTW program group and 299 days (IQR 71-365 days) in the usual care group (log rank test; $P=0.12$ ). The median total number of days at work during follow-up was 128 days (IQR 0-247 days) in the participatory RTW program group and 46 days (IQR 0-246 days) in the usual care group. In Fig. 2 the Kaplan-Meier curves for time until sustainable first RTW are presented for both groups. The crude Cox regression analysis showed a violation of the proportional hazard assumption with crossing of the survival curves at approximately 90 days follow-up. Therefore, a 


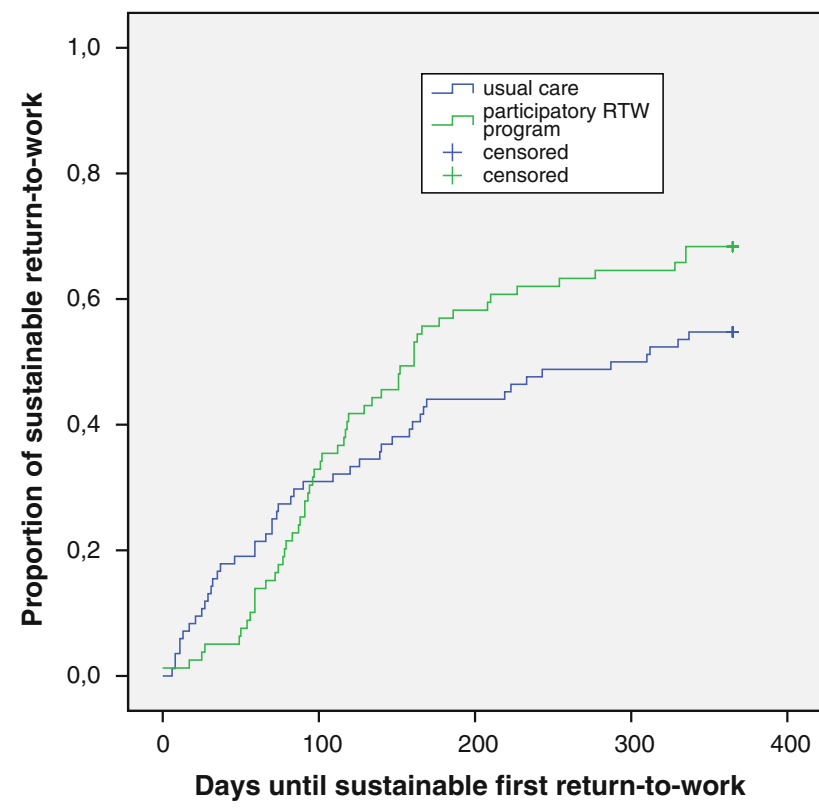

Fig. 2 Kaplan-Meier curves for sustainable first return-to-work during the 12-months follow-up for the participatory return-to-work program group and the usual care group

time-dependent covariate ( $\mathrm{T}>90$ days) was added to the Cox proportional hazards model $(P=0.011)$. To adjust for significant confounding, the baseline variables 'work schedule in last work' and 'intention to RTW despite symptoms' were included in the model (Table 2). The resulting adjusted HR ( $\mathrm{T} \leq 90$ days) was 0.76 (95\% CI $0.42-1.37 ; P=0.36$ ), and the adjusted HR ( $\mathrm{T}>90$ days) was 2.24 (95\% CI 1.28-3.94; $P=0.005$ ). The per-protocol analysis showed an adjusted HR ( $\mathrm{T} \leq 90$ days) of 0.93 (95\% CI $0.49-1.87 ; P=0.83$ ), and an adjusted HR ( $>90$ days) of 2.25 (95\% CI 1.28-3.98; $P=0.005$ ). In addition, the per-protocol analysis showed a median time until sustainable RTW of 157 days (IQR 89-365 days) in the participatory RTW program group and 330 days (IQR 87-365 days) in the usual care group (log rank test; $P=0.029$ ). Significant clustering on the level of the insurance physicians and on the level of the couples of labour experts and RTW coordinators was not found in the analyses (Table 3).

\section{Secondary Outcome Measures}

\section{Duration of Sickness Benefit}

The median claim duration until first sustainable ending of sickness benefit was 160 days (IQR 39-365 days) in the participatory RTW program group and 91 days (IQR 33-344 days) in the usual care group (Mann-Whitney U test; $P=0.14)$. The per-protocol analysis results differed slightly and showed a median duration of 168 days
(IQR 45-365 days) and 109 days (IQR 35-365 days), respectively (Mann-Whitney U test; $P=0.18$ ).

\section{Attitude, Social Influence, and Self-Efficacy (ASE) Determinants}

Table 4 presents the results of the mixed model analyses for the Attitude, Social influence, and self-Efficacy determinants, accounted for possible clustering on the level of the insurance physicians. After 3 months of follow-up both groups experienced more social influence to RTW, but developed a less positive attitude towards RTW compared to baseline. However, no statistically significant differences were found between both groups.

\section{Health-Related Outcomes}

Table 4 also presents the results on the effectiveness of the participatory RTW program on health-related outcomes, accounted for possible clustering on the level of the insurance physicians. No statistically significant differences were found between the improvements in functional status, pain intensity, and perceived health in the participatory RTW program group and the usual care group.

\section{Discussion}

\section{Main Findings}

This paper presents the effects of a newly developed participatory RTW program for temporary agency workers and unemployed workers, sick-listed due to MSD, compared to usual care. The main findings of this study are a non-significant trend towards delayed RTW in the intervention group in the first 90 days, followed by a significant advantage in RTW rate after 90 days (hazard ratio of 2.24). In addition, the median duration until sustainable first RTW was 161 days in the participatory RTW program group, compared to 299 days in the usual care group. The initial delay in RTW found in the intervention group can be due to more intensive involvement after enrolment in the new participatory RTW program. A similar finding has been described by others [31, 32]. With regard to the considerable gain in RTW rate after 90 days, this is mostly due to significant more and earlier work resumption in the intervention group from 90 days onward until the end of the 12 months follow-up. Finally, no significant differences were found with regard to the measured secondary outcomes. 
Table 3 Differences in return-to-work (RTW) between the participatory RTW program group the and usual care group

\begin{tabular}{|c|c|c|c|c|c|c|c|}
\hline \multirow[t]{2}{*}{ Adjusted model $^{\mathrm{a}}$} & & \multirow{2}{*}{$\begin{array}{l}\text { Regression } \\
\text { coefficient }\end{array}$} & \multirow[t]{2}{*}{ SE } & \multirow[t]{2}{*}{$P$ value } & \multirow[t]{2}{*}{ HR } & \multicolumn{2}{|c|}{$95 \% \mathrm{CI}$} \\
\hline & & & & & & Lower & Upper \\
\hline \multirow[t]{2}{*}{ Intervention } & $\mathrm{T} \leq 90$ days & -0.29 & 0.30 & 0.34 & 0.75 & 0.42 & 1.34 \\
\hline & $\mathrm{T}>90$ days & 0.78 & 0.28 & 0.01 & 2.19 & 1.26 & 3.80 \\
\hline \multirow[t]{2}{*}{ Adjusted for work schedule } & $\mathrm{T} \leq 90$ days & -0.23 & 0.30 & 0.44 & 0.79 & 0.44 & 1.43 \\
\hline & $\mathrm{T}>90$ days & 0.84 & 0.29 & $<0.005$ & 2.32 & 1.32 & 4.10 \\
\hline \multirow{2}{*}{$\begin{array}{l}\text { Adjusted for intention } \\
\text { to RTW despite symptoms }\end{array}$} & $\mathrm{T} \leq 90$ days & -0.33 & 0.30 & 0.27 & 0.72 & 0.40 & 1.29 \\
\hline & $\mathrm{T}>90$ days & 0.74 & 0.28 & 0.01 & 2.10 & 1.20 & 3.66 \\
\hline \multirow{2}{*}{$\begin{array}{l}\text { Adjusted for work schedule }+ \text { intention } \\
\text { to RTW despite symptoms }\end{array}$} & $\mathrm{T} \leq 90$ days & -0.27 & 0.30 & 0.36 & 0.76 & 0.42 & 1.37 \\
\hline & $\mathrm{T}>90$ days & 0.81 & 0.29 & 0.01 & 2.24 & 1.28 & 3.94 \\
\hline \multirow[t]{2}{*}{ Clustering on level insurance physician } & $\mathrm{T} \leq 90$ days & -0.30 & 0.28 & 0.42 & 0.74 & 0.35 & 1.55 \\
\hline & $\mathrm{T}>90$ days & 0.74 & 0.47 & $<0.005$ & 2.10 & 1.33 & 3.22 \\
\hline \multirow{2}{*}{$\begin{array}{l}\text { Clustering on level labour } \\
\text { expert }+ \text { RTW coordinator }\end{array}$} & $\mathrm{T} \leq 90$ days & -0.25 & 0.35 & 0.47 & 0.78 & 0.40 & 1.54 \\
\hline & $\mathrm{T}>90$ days & 0.73 & 0.26 & 0.01 & 2.10 & 1.24 & 3.48 \\
\hline
\end{tabular}

Cox proportional hazards models from the adjusted Cox regression analyses. Regression coefficients, standard errors (SE), $P$ values, hazard ratio's (HR) and 95\% confidence intervals (CI) are presented

${ }^{a}$ Results of the crude Cox regression model are not presented, due to violation of the proportional hazard assumption, i.e. crossing of the survival curves at approximately 90 days follow-up

\section{Strengths of This Study}

A strength of this study is the focus on a vulnerable group within the working population, namely sick-listed workers without an employment contract or with a flexible labour arrangement. These workers are burdened with a 'labour market handicap', with the absence of a workplace/ employer to return to when sick-listed being a major RTW obstacle $[15,16]$. Therefore, creating an actual RTW perspective by offering the possibility of a temporary (therapeutic) workplace is also an important strength of this study.

Furthermore, our primary outcome measure, i.e. sustainable first RTW, should be considered a strength of this study. First RTW is commonly used as an outcome measure for RTW interventions, but does not include possible recurrences of sickness absence shortly after work resumption. By defining sustainable RTW as RTW for at least 28 days without relapse, the results in this study can be considered more robust [33].

\section{Limitations of This Study}

A limitation of this pragmatic RCT is the absence of blinding of both the sick-listed workers and the occupational health care professionals of the SSA to the allocation outcome. Unfortunately, due to the nature of the participatory intervention program, blinding was not possible.

A second limitation is the duration of the follow-up period. The study population is characterised by a greater distance to the labour market and an increased risk for long-term work disability. To assess whether the beneficial effect of the participatory RTW program remains after the 12 months follow-up, an additional measurement after 2 years with RTW data collected from the SSA database could provide more insight and possibly increase the validity of the results found in this study.

A third limitation is the generalization of the results of this study to another context, e.g. other countries. The participatory RTW program was specifically tailored for our study population and the Dutch context in which it was implemented [15]. Application of this intervention in a different setting should be preceded by tailoring of the program, taking into account the specific characteristics of the population as well as the social, political and cultural context in which the program will be implemented and used.

\section{Comparison with Other Studies}

Findings in the international literature show that workplacebased interventions are effective in reducing sickness absence among workers with musculoskeletal disorders [34]. More specifically, participatory RTW interventions including a workplace component have shown to be effective on work-related outcomes for sick-listed employees with sub-acute low back pain, i.e. in the early stage of sickness absence [17, 35], as well as for chronic back pain patients with an advanced phase of work disability [18]. However, while the above-mentioned studies focused on regular employees, i.e. those with relative permanent 
Table 4 Results of the mixed model analyses

\begin{tabular}{|c|c|c|c|c|c|c|}
\hline & Group & Baseline & 3 months & 6 months $^{\mathrm{a}}$ & 12 months $^{\mathrm{a}}$ & $\begin{array}{l}\text { Group*Time } \\
P \text { value }\end{array}$ \\
\hline \multicolumn{7}{|l|}{ Functional status (0-100 score) (RAND-36) } \\
\hline \multirow[t]{2}{*}{ Bodily pain } & PWP & $27.7(15.9)$ & $48.8(20.2)$ & $47.4(21.4)$ & $51.4(23.9)$ & 0.22 \\
\hline & UC & $29.4(15.4)$ & $45.7(23.0)$ & $50.0(23.0)$ & $53.9(25.4)$ & \\
\hline \multirow[t]{2}{*}{ Physical functioning } & PWP & $46.0(22.1)$ & $57.3(23.4)$ & $57.6(23.2)$ & $59.4(23.6)$ & 0.73 \\
\hline & UC & $51.4(21.3)$ & $59.8(25.2)$ & $64.5(24.2)$ & $66.5(26.2)$ & \\
\hline \multirow[t]{2}{*}{ Physical role functioning } & PWP & $10.4(20.6)$ & $29.7(38.8)$ & $31.6(41.1)$ & $46.8(44.0)$ & 0.13 \\
\hline & UC & $5.1(13.3)$ & $24.7(36.7)$ & $38.3(41.7)$ & $45.4(43.6)$ & \\
\hline \multirow[t]{2}{*}{ Social functioning } & PWP & $49.4(25.4)$ & $62.9(24.0)$ & $66.6(25.1)$ & $65.9(26.0)$ & 0.72 \\
\hline & UC & $51.2(27.5)$ & $58.9(26.1)$ & $66.1(25.3)$ & $63.7(28.8)$ & \\
\hline \multicolumn{7}{|l|}{ Health status (0-100 score) (RAND-36) } \\
\hline \multirow[t]{2}{*}{ Perceived present health } & PWP & $56.3(21.8)$ & $52.4(20.1)$ & $56.6(22.1)$ & $58.5(21.5)$ & 0.70 \\
\hline & UC & $60.0(20.3)$ & $55.0(23.3)$ & $55.9(24.2)$ & $59.0(24.1)$ & \\
\hline \multirow[t]{2}{*}{ Change in health } & PWP & $31.4(25.6)$ & $41.8(26.0)$ & $48.8(28.3)$ & $58.1(29.6)$ & 0.17 \\
\hline & $\mathrm{UC}$ & $38.1(25.3)$ & $38.7(30.3)$ & $50.8(28.4)$ & $56.3(31.3)$ & \\
\hline \multicolumn{7}{|l|}{ Pain intensity (1-10 score) (Von Korff) } \\
\hline \multirow[t]{2}{*}{ Back pain } & PWP & $7.2(1.9)$ & $6.0(2.2)$ & $5.6(2.3)$ & $5.4(2.6)$ & 0.92 \\
\hline & $\mathrm{UC}$ & $6.8(2.0)$ & $5.6(2.5)$ & $5.0(2.8)$ & $4.9(2.8)$ & \\
\hline \multirow[t]{2}{*}{ Neck pain } & PWP & $7.5(1.5)$ & $5.3(2.3)$ & $4.4(3.0)$ & $4.4(3.2)$ & 0.52 \\
\hline & UC & $6.5(1.9)$ & $5.3(2.9)$ & $4.0(3.2)$ & $4.2(3.1)$ & \\
\hline \multirow[t]{2}{*}{ Other pain } & PWP & $6.7(1.8)$ & $6.0(2.2)$ & $5.0(2.7)$ & $4.9(3.0)$ & 0.89 \\
\hline & $\mathrm{UC}$ & $6.2(1.9)$ & $5.7(2.3)$ & $5.1(2.5)$ & $4.7(3.0)$ & \\
\hline \multicolumn{7}{|c|}{ Attitude, social influence, self-efficacy determinants } \\
\hline \multirow[t]{2}{*}{ Attitude to RTW ( -5 to 12 ) } & PWP & $5.13(4.27)$ & $3.41(5.21)$ & - & - & 0.18 \\
\hline & $\mathrm{UC}$ & $4.87(3.96)$ & $1.92(5.81)$ & - & - & \\
\hline \multirow[t]{2}{*}{ Social influence to RTW ( -26 to 18 ) } & PWP & $-5.16(8.72)$ & $-2.13(9.26)$ & - & - & 0.16 \\
\hline & $\mathrm{UC}$ & $-3.39(8.89)$ & $-2.59(9.20)$ & - & - & \\
\hline \multirow[t]{2}{*}{ Self-efficacy to RTW ( -4 to 4 ) } & PWP & $0.42(2.43)$ & $0.44(2.12)$ & - & - & 0.79 \\
\hline & UC & $0.06(2.26)$ & $0.19(2.33)$ & - & - & \\
\hline \multirow[t]{2}{*}{ Intention to RTW despite symptoms (1-5) } & PWP & $3.46(1.10)$ & $3.65(1.24)$ & - & - & 0.32 \\
\hline & UC & $3.05(1.19)$ & $3.53(1.39)$ & - & - & \\
\hline Response rate questionnaires (\%) & & 100 & 85.3 & 77.9 & 81.6 & \\
\hline
\end{tabular}

Differences in health-related outcomes, and the attitude, social influence, and self-efficacy determinants between the participatory RTW program group (PWP) and usual care group (UC), accounted for possible clustering on the level of the insurance physician. Unless indicated otherwise the observed mean and standard deviation are presented

a Attitude, social influence, and self-efficacy determinants were only measured at baseline and 3 months

employment relationships, this study shows that a participatory RTW intervention with the possibility of a suitable (therapeutic) workplace is also effective on RTW for a more vulnerable group within the working population, i.e. sicklisted workers who have no (longer) an employer/workplace to return to. In addition, our study findings show that the participatory RTW program can also be applied for workers with all types of MSD, not merely for workers with low back pain.

The absence of beneficial or adverse effects on secondary health-related outcomes in this study is in line with recent findings of Lambeek and colleagues [18], and supports the work disability paradigm, i.e. recovery of health is not a necessary precondition for work resumption. The discrepancy between work-related outcomes and health outcomes has also been reported by others [34]. A possible explanation for this is the focus of the intervention on reducing barriers for RTW and not on symptomatic recovery from MSDs.

In occupational health care research there is an increasing awareness of the importance of behavioural determinants in the field of RTW research and intervention development [36-38]. Work attitude, social support, selfefficacy, and intention to RTW all have been associated 
with time to RTW. In our study no statistically significant differences were found between both groups for changes in Attitude, Social support, and self-Efficacy (ASE) determinants. However, the ASE determinants were only measured at baseline and after 3 months of follow-up. In view of the significant gain in more rapid RTW after 90 days, it is possible that potentially favourable effects on behavioural determinants were present at a later stage during follow-up, but were not measured. Nevertheless, in line with the findings of van Oostrom and colleagues [38], the variable 'intention to RTW despite symptoms' showed to be a significant confounder for sustainable first RTW in the Cox regression analysis.

\section{Implications for Practice}

With an eminent earlier work resumption (intention-totreat: median of 138 days; per-protocol: median of 173 days) during one-year of follow-up, the newly developed participatory RTW program seems to be a promising intervention to enhance work resumption and reduce work disability among temporary agency workers and unemployed workers, sick-listed due to MSD. However, although not statistically significant, the new RTW program had a negative impact on sickness benefit duration (intention-to-treat: median of 69 days; per-protocol: median of 59 days). This was mainly due to the fact that in most cases the therapeutic workplaces were offered with ongoing sickness benefit, i.e. the total number of days working in these temporary workplaces represented $95 \%$ of the difference in total benefit duration between both groups. However, in our opinion, the gains in higher RTW rate and earlier RTW may counterbalance this added cost burden by enhancing social participation of vulnerable workers [39], and by generating an economic benefit in terms of productivity gain. Cost-effectiveness and costbenefit analyses will be conducted to evaluate whether the effects indeed counterbalance the costs. Moreover, these results will be essential to convince policy makers that implementation of the new RTW program is a worthwhile and necessary investment to achieve a sustainable contribution of vulnerable workers to the labour force. This approach is supported by a recent study showing that application of work interventions and less strict compensation policies to be eligible for long-term benefits contributed to sustainable RTW [40]. Nevertheless, due to the relatively short follow-up in this study, our findings should be confirmed in future studies with a longer follow-up. Another possibility could be offering subsidised (temporary) workplaces. This kind of arrangement already exists in the Netherlands for young disabled workers [41]. One could argue that such temporary arrangements can be extended to other groups of vulnerable workers within the framework of an active labour market policy.

Furthermore, in our study the RTW coordinator played a key role to guarantee (perceived) safety and equality among all stakeholders and active involvement during the making of the consensus-based RTW plan. A systematic review also showed that an important key element in RTW interventions is the active involvement of an independent RTW coordinator [42]. For successful implementation we, therefore, recommend the use of a RTW coordinator competency profile, in line with the recommendation of Pransky and colleagues [43], who stated that identification of a core set of essential RTW coordinator competencies is essential.

Acknowledgments We would like to thank all the participating occupational health care professionals at the Dutch National Social Security Agency for carrying out the participatory RTW program, and the staff and management at the Social Security Agency for their support during the study and their contribution to the implementation of the participatory RTW program. We also thank Lambèrt Balvers for his support as project manager, Jacqueline Muller and Marion Evers for their unrestrained dedication to find suitable temporary workplaces for the participants, Sietske Tamminga for her help with recruiting workers, and Karlijn van Beurden for her help with recruiting workers and data entry. This study is financially supported by the Dutch Institute for Employee Benefit schemes.

Conflict of interest The author(s) declare that they have no competing interests.

Open Access This article is distributed under the terms of the Creative Commons Attribution Noncommercial License which permits any noncommercial use, distribution, and reproduction in any medium, provided the original author(s) and source are credited.

\section{References}

1. Henderson M, Glozier N, Holland EK. Long term sickness is caused by common conditions and needs managing. BMJ. 2005; 330(7495):802-3.

2. Anema JR, van der Beek AJ. Medically certified sickness absence. BMJ. 2008;337:a1174.

3. Department of Health. Choosing health: making healthier choices easier (Public Health White Paper). London: DH; 2004.

4. Watson PJ, Booker CK, Moores L, Main CJ. Returning the chronically unemployed with low back pain to employment. Eur J Pain. 2004;8(4):359-69.

5. Audhoe SS, Hoving JL, Sluiter JK, Frings-Dresen MH. Vocational interventions for unemployed: effects on work participation and mental distress. A systematic review. J Occup Rehabil. 2010; 20(1):1-13.

6. Brodsky MM. Labor market flexibility: a changing international perspective. Mon Labor Rev. 1994;117(11):53-60.

7. Benach J, Gimeno D, Benavides FG, Martínez JM, Del Mar Torné M. Types of employment and health in the European Union: changes from 1995 to 2000. Eur J Public Health. 2004; 14(3):314-21.

8. Benach J, Muntaner C. Precarious employment and health: developing a research agenda. J Epidemiol Community Health. 2007;61(4):276-7. 
9. Benach J, Benavides FG, Platt S, Diez-Roux A, Muntaner C. The health-damaging potential of new types of flexible employment: a challenge for public health researchers. Am J Public Health. 2000;90(8):1316-7.

10. Jin RL, Shah CP, Svoboda TJ. The impact of unemployment on health: a review of the evidence. CMAJ. 1995;153(5):529-40.

11. Benach J, Amable M, Muntander C, Benavides F. The consequences of flexible work for health: are we looking at the right place? J Epidemiol Community Health. 2002;56(6):405-6.

12. Quinlan M, Mayhew C, Bohle P. The global expansion of precarious employment, work disorganization, and consequences for occupational health: a review of recent research. Int $\mathbf{J}$ Health Serv. 2001;31(2):335-414.

13. Arents MR, Dorenbos I, Vogelaar B, Vrijhof B, Landheer W. Aard en oorzaken ziekteverzuim uitzendbranche [Nature and causes of sickness absence among temporary agency workers]. Rotterdam: ECORYS-NEI; 2003.

14. Reijenga FA, Veerman TJ, van den Berg N. Onderzoek evaluatie wet verbetering poortwachter [Evaluation of the Improved Gatekeeper Act]. Leiden: Astri; 2006.

15. Vermeulen SJ, Anema JR, Schellart AJM, van Mechelen W, van der Beek AJ. Intervention mapping for development of a participatory return-to-work intervention for temporary agency workers and unemployed workers sick-listed due to musculoskeletal disorders. BMC Public Health. 2009;9:216.

16. Vermeulen SJ, Tamminga SJ, Schellart AJM, Ybema JF, Anema JR. Return-to-work interventions for sick-listed workers without an employment contract-what works? BMC Public Health. 2009; 9:232.

17. Anema JR, Steenstra IA, Bongers PM, de Vet HC, Knol DL, Loisel $\mathrm{P}$, et al. Multidisciplinary rehabilitation for sub acute low back pain: graded activity or workplace intervention or both? A randomized controlled trial. Spine. 2007;32(3):291-8.

18. Lambeek LC, van Mechelen W, Knol DL, Loisel P, Anema JR. Randomised controlled trial of integrated care to reduce disability from chronic low back pain in working and private life. BMJ. 2010;340:c1035.

19. Vermeulen SJ, Anema JR, Schellart AJM, van Mechelen W, van der Beek AJ. Cost-effectiveness of a participatory return-to-work intervention for temporary agency workers and unemployed workers sick-listed due to musculoskeletal disorders: design of a randomised controlled trial. BMC Musculoskelet Disord. 2010;11:60.

20. Cheadle A, Franklin G, Wolfhagen C, Savarino J, Liu PY, Salley C, et al. Factors influencing the duration of work-related disability: a population-based study of Washington State workers' compensation. Am J Public Health. 1994;84(2):190-6.

21. Bartley M, Sacker A, Clarke P. Employment status, employment conditions, and limiting illness: prospective evidence from the British household panel survey 1991-2001. J Epidemiol Community Health. 2004;58(6):501-6.

22. Abásolo L, Carmona L, Lajas C, Candelas G, Blanco M, Loza E, et al. Prognostic factors in short-term disability due to musculoskeletal disorders. Arthr Rheum. 2008;59(4):489-96.

23. De Zwart BC, Broersen JP, van der Beek AJ, Frings-Dresen MH, van Dijk FJ. Occupational classification according to work demands: an evaluation study. Int J Occup Med Environ Health. 1997;10(3):283-95.

24. Steenstra IA, Verbeek JH, Heymans MW, Bongers PM. Prognostic factors for duration of sick leave in patients sick listed with acute low back pain: a systematic review of the literature. Occup Environ Med. 2005;62(12):851-60.

25. Von Korff M, Ormel J, Keefe FJ, Dworkin SF. Grading the severity of chronic pain. Pain. 1992;50(2):133-49.

26. Ware JE Jr, Sherbourne CD. The MOS 36-item short-form health survey (SF-36). I. Conceptual framework and item selection. Med Care. 1992;30(6):473-83.
27. Aaronson NK, Muller M, Cohen PD, Essink-Bot ML, Fekkes M, Sanderman R, et al. Translation, validation, and norming of the Dutch language version of the SF-36 Health Survey in community and chronic disease populations. J Clin Epidemiol. 1998;51(11): $1055-68$.

28. Kraaimaat FW, Bakker A, Evers AWM. Pijncoping-strategieën bij chronische pijnpatiënten: de ontwikkeling van de Pijn-Coping-Inventarisatielijst (PCI) [Pain coping strategies in chronic pain patients: development of the Pain-Coping-Inventory questionnaire (PCI)]. Gedragstherapie. 1997;30:185-201.

29. van Oostrom SH, Anema JR, Terluin B, de Vet HCW, Knol DL, van Mechelen W. Cost-effectiveness of a workplace intervention for sick-listed employees with common mental disorders: design of a randomized controlled trial. BMC Public Health. 2008;8:12.

30. Gutierrez RG. Parametric frailty and shared frailty survival models. Stata J. 2002;2(1):22-44.

31. Staal JB, Hlobil H, Twisk JW, Smid T, Köke AJ, van Mechelen W. Graded activity for low back pain in occupational health care: a randomized, controlled trial. Ann Intern Med. 2004;140(2):77-84.

32. Hlobil H, Staal JB, Twisk J, Köke A, Ariëns G, Smid T, et al. The effects of a graded activity intervention for low back pain in occupational health on sick leave, functional status and pain: 12-months results of a randomized controlled trial. J Occup Rehabil. 2005;15(4):569-80.

33. Baldwin ML, Butler RJ. Upper extremity disorders in the workplace: costs and outcomes beyond the first return to work. J Occup Rehabil. 2006;16(3):303-23.

34. van Oostrom SH, Driessen MT, de Vet HCW, Franche RL, Schonstein E, Loisel P, et al. Workplace interventions for preventing work disability. Cochrane Librar. 2009;15.

35. Loisel P, Abenhaim L, Durand P, Esdaile JM, Suissa S, Gosselin $\mathrm{L}$, et al. A population-based, randomized clinical trial on back pain management. Spine. 1997;22(24):2911-8.

36. Krause N, Frank JW, Dasinger LK, Sullivan TJ, Sinclair SJ. Determinants of duration of disability and return-to-work after work-related injury and illness: challenges for future research. Am J Ind Med. 2001;40(4):464-84.

37. Brouwer S, Krol B, Reneman MF, Bültmann U, Franche RL, van der Klink JJ, et al. Behavorial determinants as predictors of return to work after long-term sickness absence: application of the theory of planned behaviour. J Occup Rehabil. 2009;19(2):166-74.

38. van Oostrom SH, van Mechelen W, Terluin B, de Vet HCW, Knol DL, Anema JR. A workplace intervention for sick-listed employees with distress: results of a randomized controlled trial. Occup Environ Med. 2010;67(9):596-602.

39. Schuring M, Mackenbach J, Voorham T, Burdorff A. The effect of re-employment on perceived health. J Epidemiol Community Health. 30 Aug 2010 [Epub ahead of print].

40. Anema JR, Schellart AJM, Cassidy JD, Loisel P, Veerman TJ, van der Beek AJ. Can cross country differences in return-to-work after chronic occupational back pain be explained? An exploratory analysis on disability policies in a six country study. J Occup Rehabil. 2009;19(4):419-26.

41. Reijenga FA, Prins R. Bruggen bouwen naar de arbeidsmarkt voor jongeren met een beperking [Building bridges towards the labour market for young disabled persons]. Leiden: Astri; 2008.

42. Franche RL, Cullen K, Clarke J, Irvin E, Sinclair S, Frank J. The Institute for Work and Health (IWH) workplace-based RTW intervention literature review research team: Workplace-based return-to-work interventions: a systematic review of the literature. J Occup Rehabil. 2005;15(4):607-31.

43. Pransky G, Shaw WS, Loisel P, Hong QN, Désorcy B. Development and validation of competencies for return to work coordinators. J Occup Rehabil. 2010;20(1):41-8. 